REVISTA PERUANA DE INVESTIGACIÓN EDUCATIVA $2013, \mathrm{No} .5$, p p.124-155

Efectos de la composición social y la repetición sobre el rendimiento escolar en competencia lectora

Effects of social composition and grade repetition on student performance in reading

\author{
Luis Muelle \\ Consultor en educación \\ luismuelle@yahoo.com
}

Recibido: 05.07.2013

Revisado: 02.09.2013

Aprobado: 25.11.2013 


\section{Resumen}

Este trabajo explora las relaciones y efectos que existen entre los antecedentes socioeconómicos del alumno, la composición social de las escuelas y la repetición de grado en el rendimiento de los alumnos. Utilizando los resultados de la prueba en Competencia Lectora de la evaluación PISA 2009 aplicada en el Perú, el interés se focaliza en los probables beneficios que puede aportar la repetición como práctica escolar para mejorar los aprendizajes.

Aunque los datos no permiten inferencias causales, los análisis efectuados sugieren que los alumnos que han conocido la repetición en su carrera escolar obtienen sistemáticamente rendimiento escolares más bajos que sus compañeros no-repetidores. Se ha constatado, también, que las escuelas favorecidas socialmente tienen altos rendimientos asociados a una baja repetición, mientras que las escuelas desfavorecidas tienen más alta repetición y más bajos rendimientos. Además, se ha identificado tres ventajas producidas por efectos de los antecedentes socioeconómicos de los alumnos. La primera es para aquellos que provienen de hogares favorecidos socialmente, quienes obtienen en todos los casos mejores puntajes conforme su capital social se acrecienta. Una segunda ventaja viene de la textura social de la escuela frecuentada por un público escolar favorecido, que también se ve bonificado con mayores puntajes. En fin, estudiar en una escuela favorecida socialmente protege mejor de la repetición que el propio antecedente socioeconómico del alumno. Esta exploración de variables que se asocian con el rendimiento del alumno, así como los efectos de la repetición, brindan información que puede contribuir al debate sobre la pertinencia de esta práctica escolar.

Palabras clave: rendimiento escolar, composición social, competencia lectora, repetición

\section{Abstract}

In this article I explore the relationships and effects between the students' socio-economic background, the social composition of schools and the grade repetition in students' performances. Using the results of PISA 2009 on reading competence in Peru, I focus on the likely benefits of repetition as a school practice to improve learning.

Although it is not possible to establish causal inferences in the data, the analyses suggest that students who repeat in their school career obtain a lower performance than their peers who do not. I have also found that social advantaged schools have a high-performance associated to a low repetition 
while disadvantaged schools have high repetition and low performances. In addition, I have identified three advantages generated by the students' socioeconomic background: 1) those coming from socially advantaged homes obtain better scores as their social capital increases, 2) those who attend a school with a social advantaged population also benefit with better scores, and 3) studying in a socially favorable school protects against repetition better than the student's own social background. This exploration of variables associated with student achievement, as well as the effects of repetition, offer new insights to the debate about the benefit of this school practice.

Keywords: Academic achievement, social composition, reading competence, grade repetition 


\title{
Efectos de la composición social y la repetición sobre el rendimiento escolar en competencia lectora
}

\section{Introducción}

\begin{abstract}
A ctualmente, se asiste a una profusión de evaluaciones de los aprendizajes de alumnos que se llevan a cabo en una variedad de países por las organizaciones internacionales. A este respecto, son conocidos los esfuerzos de los programas TIMSS, Pirls, Confemen, Sacmeq, Llece. Sin embargo, por su envergadura, merecen mención particular las evaluaciones llevadas a cabo por la OCDE sobre la calidad y equidad de la educación, con el mundialmente conocido Programa Internacional para la Evaluación de Alumnos (PISA), que data desde el año 2000 y en cuya versión de 2009 participaron 65 países. Este programa presenta el perfil de conocimientos y habilidades de los alumnos de quince años en competencia lectora, matemática y científica, así como los indicadores contextuales que relacionan el rendimiento logrado en estas disciplinas con sus propias características personales, familiares y con aquellas del centro escolar que frecuentan.
\end{abstract}

Estos estudios llaman poderosamente la atención sobre la repetición de grado como un factor permanentemente negativo asociado con el rendimiento escolar, pues se constata que, en países en los que los alumnos son promovidos automáticamente de grado, su rendimiento no es por eso inferior al de los estudiantes de aquellos países en los que existe la repetición. Al contrario, ciertos países que practican severa y masivamente la repetición muestran rendimientos muy modestos. La OCDE propone, por eso, la eliminación de la repetición de grado por constituir una política costosa e ineficaz, que no garantiza la mejora de los resultados educativos (2012a). Igualmente, para América Latina, el Llece -como resultado de su estudio de factores asociados con el rendimiento- plantea la eliminación de la repetición, pues ella se relaciona negativamente con el aprendizaje y tiene [sic] efectos perversos $y$ costosos (Llece, 2010, p. 18).

En el mismo sentido, se levantan otras voces en contra de esta práctica educativa. Tal es el caso de países francófonos del África (Pasec/Confemen, 2005), en los que -después de realizar un seguimiento de cohortes de alumnos para estudiar la repetición en primaria- los evaluadores constatan que, a lo largo de su trayectoria escolar, los estudiantes repetidores progresan menos en matemáticas y en lectura que sus compañeros de la misma cohorte generacional. La repetición presenta una falsa impresión de eficacia, que provoca una especie de espejismo en las escuelas africanas. Asimismo, en África, pero esta vez en los países anglófonos agrupados en el consorcio Sacmeq (Southern and Eastern África Consortium for Monitoring Education Quality), 
se reportan serios problemas de repetición y de rendimiento. En efecto, luego de estudiar los resultados de las pruebas de lectura y matemáticas del sexto grado de primaria, existe la convicción de que los alumnos repetidores no logran alcanzar mejores puntajes que sus compañeros no-repetidores en ninguno de los quince países del Consorcio (Ikeda, 2005).

La repetición ha provocado y provoca un debate continuo en el seno de la comunidad educativa y tanto sus defensores como sus detractores no cesan de afrontar sus datos y argumentos. La disponibilidad pública de datos provenientes del estudio PISA 2009 ofrece la ocasión para abordar estos aspectos, pues permite enriquecer este debate con el aporte de información sobre las características del contexto personal, familiar, escolar y social del alumno en relación con el resultado de una prueba de comprensión lectora. Ayudar a este debate es el propósito del presente estudio, que focaliza su interés en los efectos de la repetición en términos de rendimiento escolar en el caso del Perú, país que ha participado en el programa PISA $2009^{1}$.

\section{Objetivos y límites del estudio}

Se ha señalado la prevalencia de la repetición de grado escolar como factor negativamente relacionado con el bajo rendimiento escolar. Aunque el propósito de esta medida es brindar a los alumnos repetidores mayores oportunidades de aprendizaje, los rendimientos que se constatan en las evaluaciones no parecen confirmar los beneficios de esta práctica escolar. Desde luego, estos resultados no abogan en favor de la repetición, pero tampoco permiten concluir directamente su ineficacia.

En PISA 2009, el rendimiento es medido a través de la competencia lectora, que consiste en comprender, utilizar, reflexionar e interesarse por los textos escritos para desarrollar el conocimiento y potencial personales, y participar en la sociedad (OCDE, 2010a). El presente estudio examina la distribución de este desempeño obtenido por los alumnos en la prueba de competencia lectora a partir de la base de datos de PISA 2009 correspondiente al Perú².

1 El Ministerio de Educación del Perú lleva a cabo regularmente evaluaciones de alumnos desde 2007. Estas evaluaciones se refieren a Matemáticas y Comprensión de Textos en 2 do y 4 to grado de primaria (Educación Bilingüe). Un análisis de las evaluaciones tanto nacionales como internacionales en las que participa el país es presentado y discutido ampliamente por Cueto (2007) y por Miranda (2008). También, se puede consultar el sitio web Unidad de Medición de Calidad Educativa (2012).

128 I 2 Esta base de datos se encuentra disponible en OECD (s.f.). 
El primer objetivo es medir la ocurrencia de diferencias significativas entre los alumnos repetidores y aquellos que no repiten. En segundo lugar, a través de modelos multivariados, se pretende analizar el impacto de los antecedentes socioeconómicos de los alumnos, de la composición social de la escuela y de la repetición escolar sobre el rendimiento escolar. Ello tiene el propósito de investigar la posible relación y la magnitud del efecto de estos tres factores.

Se debe señalar algunos límites de este estudio. Para empezar, los análisis que se presentan reflejan las asociaciones entre variables y no tienen como propósito proponer causalidades, pues solamente los estudios longitudinales y los diseños experimentales proporcionan argumentos válidos en estos términos. Además, la muestra ha sido diseñada para presentar datos válidos y representativos sobre las características de los alumnos, más que sobre aquellas de las escuelas. Asimismo, es importante señalar que el rendimiento de los alumnos registrados por la prueba PISA no puede ser considerado solamente como el producto de las condiciones actuales de escolaridad en el país.

\section{Metodología}

Además de pasar la prueba de competencias en Comprensión Lectora, Matemáticas y Ciencias, los alumnos han respondido a un cuestionario sobre sus antecedentes personales y familiares, sus hábitos de estudio, su actitud hacia la lectura, así como su nivel de implicación y motivación. En paralelo, los directores de los centros escolares han completado un cuestionario sobre sus centros, lo cual incluye datos sobre su situación demográfica y una evaluación de la calidad de su ambiente de aprendizaje.

En el Perú, la fecha de evaluación se aplicó entre el 19 y el 20 de agosto de 2009 a una muestra de alumnos de escuelas del nivel secundario o su equivalente (Secundaria de EBR, Secundaria para Adultos, Centros de Educación Básica Alternativa - CEBA), nacidos entre el 1 de mayo de 1993 y el 30 de abril de 1994. Han participado 240 escuelas y 5.985 estudiantes. La distribución por grados de secundaria fue la siguiente: 1 er grado $(4 \%), 2$ do grado $(8,9 \%), 3 e r$ grado $(17,1 \%)$, 4 to grado $(44,6 \%)$, 5 to grado $(25,4 \%)$. La prueba fue monitoreada por el Ministerio de Educación. Cabe señalar que 73\% de la población de 15 años asiste al sistema educativo (Perú-Min. Educación, 2010)³.

3 Las correspondencias entre la edad de ingreso, la edad actual y el grado cursado actualmente por los alumnos puede ser una fuente valiosa para analizar el retraso y la repetición escolar en futuros trabajos. 


\section{El puntaje en Competencia Lectora y el uso de los valores plausibles}

El rendimiento escolar se mide a través de los puntajes obtenidos por los alumnos en la prueba aplicada en el área de Competencia Lectora. Debido al diseño muestral, la encuesta tiene errores de medida, que son calculados a través de réplicas de las ponderaciones con el método BRR (balanced repeated replicate weights, en inglés). Cabe anotar que las mismas que son de uso obligatorio para todo análisis.

Para los puntajes, se utilizan los llamados "valores plausibles», que atribuyen cinco de estos valores por cada puntaje obtenido por el alumno en el test, pues no existe un valor único. Los valores plausibles son números aleatorios extraídos de la distribución teórica de los puntajes de las pruebas, que podrían asignarse razonablemente a cada estudiante en el resultado de su competencia, según la Teoría de Respuesta al Ítem (Ítem Response Theory - IRT). Por convención y arbitrariamente, los valores plausibles tienden a tener un promedio estandarizado de 500 puntos y una desviación estándar de 100 puntos. Cada promedio es calculado como una combinación simultánea del peso de cada alumno en la encuesta y cada uno de sus cinco valores plausibles.

Aunque el propósito de la encuesta PISA 2009 no ha sido estudiar la repetición, en el protocolo, hay preguntas que permiten abordar este problema. Así, la variable repetición ha sido aquí construida a partir de las respuestas a tres preguntas sobre el historial escolar de cada estudiante: repetición en la escuela primaria (ISCED1), repetición en la educación secundaria inferior (ISCED2) y repetición en la secundaria superior (ISCED3). Si el alumno responde afirmativamente por lo menos una vez a cualquiera de estas preguntas, el alumno es considerado como repetidor; en caso contrario, este será un alumno no-repetidor ${ }^{4}$.

\section{El índice de antecedentes socioeconómicos (ESCS)}

Las respuestas a los cuestionarios de contexto producen un variado conjunto de índices. El más importante -y que será utilizado en este estudio- es el llamado índice de estatus económico, social y cultural (ESCS, por sus siglas en inglés), que ha sido construido a partir de tres índices simples: el máximo nivel ocupacional de los padres (Hisei), el máximo nivel educativo de los padres en años de escolarización según la terminología de la Unesco/Isced/ CINE (Pared) y el índice de posesiones en el hogar (Homepos). Cada uno de

4 Los valores ausentes (missing values, en inglés) de esta variable representan 1,2\% del total. 
ellos contiene a su vez un conjunto de ítems, cuya construcción se ha realizado a través de la metodología de componentes principales. Para cumplir con uno de los objetivos de este estudio, se ha construido además la variable auxiliar Mu_Escuela que es el promedio del índice ESCS correspondiente a cada escuela. Este indicador permite una aproximación de lo que se ha convenido llamar la composición social de la escuela, es decir, la presencia de alumnos con distinto antecedente social que asiste a la misma institución educativa. Conviene indicar que, a pesar de la ventaja de su disponibilidad, la utilización de este y otros índices propuestos por PISA, estos pueden ser objeto de afinamiento en su construcción y alcances (Guadalupe y Villanueva, 2013).

\section{Los niveles de Competencia Lectora}

El puntaje logrado en el test se alinea a una escala de Competencia Lectora que sirve para interpretar el significado de los puntajes de los alumnos en términos sustantivos. Esta escala se divide en seis niveles; cada uno tiene un rango de dificultad particular que describe los tipos de habilidades y conocimientos necesarios para completar con éxito las tareas del nivel correspondiente de las preguntas. La escala va desde el Nivel 1 -que tiene dos sub niveles a y b-, que es el nivel más bajo; seguido del Nivel 2, del Nivel 3 y, así, sucesivamente hasta el Nivel 6 . A continuación, se presenta una síntesis de estos seis niveles de competencia lectora y sus fronteras de puntaje entre paréntesis:

- $\quad$ Nivel 6 (698 puntos) - El lector realiza múltiples deducciones, comparaciones y contrastes, detallados y precisos.

- Nivel 5 (626 puntos) - El lector localiza y organiza varias piezas de información poco prominente, y deduce qué datos del texto son relevantes.

- Nivel 4 (553 puntos) - El lector localiza y organiza varias piezas de información poco prominentes.

- Nivel 3 (480 puntos) - El lector localiza y reconoce la relación entre varias piezas de información e integra diversas partes del texto con el fin de identificar una idea principal, comprender su relación y significado.

- Nivel 2 (407 puntos) - El lector localiza una o más piezas de información, reconoce la idea principal del texto, comprende relaciones y realiza deducciones de bajo nivel.

- Nivel 1a (335 puntos) - El lector localiza una o más piezas independientes de información; reconoce el tema principal y realiza una sencilla conexión entre información en el texto y conocimientos comunes cotidianos. 
- Nivel 1b (262) - La información en conflicto es mínima y el lector localiza una única pieza de información explícita en un texto breve, sintácticamente sencillo, con un contexto y tipo de textos conocidos (como una narración o listado sencillos).

\section{El método de exploración y análisis}

En todos los cálculos se han utilizado los puntajes promedios a partir de los valores plausibles y sus respectivas réplicas. En los análisis, se ha respetado -en todos los casos- la ponderación de la muestra como recurso obligatorio para asegurar la representatividad de los alumnos. En este estudio, no se ha aplicado ninguna imputación de remplazo a las variables continuas, tales como los puntajes e índices. Cuando la repetición es abordada como variable dependiente, por su carácter binario, se ha recurrido a métodos logísticos. Como es usual, para verificar la dispersión e inferir hipótesis sobre la población a partir de la muestra, se presentan los errores estándares.

\section{Justificación y matices de la repetición}

En el informe anual de United Nationes Educational, Scientific, and Cultural Organization -Unesco- (2012) dedicado a la repetición, se define al repetidor como el alumno que no logra ser promovido al siguiente grado o no logra finalizar un programa educativo, y debe cursar el mismo grado en el siguiente año escolar. Constata que, a escala mundial, el acceso a la enseñanza se ha ampliado de forma significativa, pero este avance se ve limitado por altas tasas de repetición, la salida prematura de la escuela por parte de los educandos y los bajos niveles de logro. Las causas que originan la repetición son variadas y difíciles de identificar. Así, ella puede ser el resultado de la asistencia irregular a la escuela por periodos de duración variada, de la edad no adecuada al grado que cursa el alumno, de la falta de oportunidades de aprendizaje en el entorno local, pero, sobre todo, de las propias dificultades de aprendizaje desarrolladas por el alumno cuando obtiene calificaciones insuficientes.

Desde hace dos décadas, Schielfelbein y Wolff (1993) señalaban la importancia y, al mismo tiempo, la subestimación de la repetición en las estadísticas oficiales en la mayoría de los países latinoamericanos. Señalaban que esto era efecto de (a) profesores que deciden la repetición de sus alumnos a pesar de que estos tienen calificaciones suficientes para ser promovidos; (b) estudiantes que abandonan el sistema escolar antes de finalizar el año 
escolar y que son registrados como desertores, pero que vuelven a la escuela al año siguiente y al mismo grado; (c) docentes que carecen de información para identificar a los alumnos que asistieron al mismo grado el año anterior d) estudiantes repetidores que, a menudo, se niegan a registrarse como tales.

La práctica de la repetición de grado se inspira frecuentemente en el convencimiento de que un año adicional en el mismo grado brindará a los alumnos con dificultades una oportunidad para fortalecer el dominio de los contenidos del curso y los preparará para tener un mejor desempeño en los grados posteriores. Los que apoyan las políticas de repetición afirman que mantener altos estándares también es importante para las escuelas ${ }^{5}$. Otros sistemas, sin embargo, adoptan la promoción automática de grado, pues cuestionan la repetición por los impactos negativos que tiene en los alumnos, en la medida que afecta su autoestima y motivación académica, así como el hecho de que les genera la impresión de que son malos estudiantes. Esta política se basa en la convicción de que la promoción mantiene motivados a los alumnos y que estos progresarán mejor en su escolaridad gracias a que estarán expuestos a nuevos contenidos (Brophy, 2006).

Después de amplios trabajos sobre la repetición, Perrenoud (1996) concluye que, cada vez que hay debate sobre las ventajas y desventajas de esta, se corre el riesgo de centrarse en los síntomas en lugar de hacerlo sobre las causas. La repetición de grado sería solo un indicador incierto de las desigualdades de aprendizaje y proponer su eliminación es una condición necesaria, pero no suficiente para elevar la calidad de los aprendizajes. Esto equivaldría a pensar que "romper el termómetro hace bajar la fiebre».

Por su parte, Torres (2000) afirma que la repetición es una solución interna que ha encontrado el sistema escolar para lidiar con el problema del noaprendizaje o de la mala calidad del mismo y juzga que, junto con la pobreza, aparece como el primer paso hacia -y el mejor predictor de- la deserción escolar:

Pedagógicamente la repetición se basa en una serie de premisas equivocadas: que el estudiante que no aprendió o no aprendió suficiente, aprenderá si toma exactamente el mismo camino por segunda vez, el camino que le hizo fracasar la primera vez; que nada se aprendió a lo largo del proceso y que, por tanto, es necesario empezar todo de nuevo desde el inicio; que el conocimiento y el aprendizaje operan en una dimensión lineal, siguen rutas fijas, y son el resultado de la ejercitación repetitiva. Socialmente, la repetición refuerza el círculo vicioso de las bajas expectativas, el bajo rendimiento, la baja autoestima y el fracaso escolar. Los padres de fami- 
lia interpretan las bajas calificaciones de sus hijos como una señal de su incapacidad para aprender. La repetición, de este modo, refuerza las peores expectativas de los padres respecto de sus hijos, su futuro y su propia condición familiar. La repetición, en definitiva, sólo atrae más repetición (Torres, 2000, p.11).

Además, algunos responsables de la educación tienden a considerarla como un problema externo a la escuela, generado en la familia y alimentado a veces por el propio alumno, por lo cual habría que buscar una solución fuera del sistema escolar. Por eso, para García-Huidobro (2001), resulta explicable que algunos padres de familia acepten fácilmente la repetición como una benévola segunda oportunidad ofrecida para aquellos hijos amenazados por el fracaso escolar. Este autor agrega que algunos segmentos de la comunidad educativa tienden a concebirla como un hecho natural inherente y hasta inevitable de la vida escolar.

Por su parte, Miranda (2011, p. 23) anota que la repetición no permite que los alumnos logren un mejor resultado, pues el sistema educativo no se responsabiliza por este problema ni desencadena los mecanismos necesarios y pertinentes para garantizar mejoras en el tramo que se repite, de modo que:

[...] es el estudiante quien asume toda la carga, pues no sólo siente una mayor presión del entorno, sino también se incrementa su costo de oportunidad para continuar estudiando, sobre todo en aquellos estudiantes que provienen de sectores más desfavorecidos. En efecto, esta manera de ver las cosas, que sitúa la carga del problema del fracaso escolar en los estudiantes, también se traduce en el casi unánime rechazo de los docentes y algunas autoridades a la introducción de políticas de promoción automática. Las concepciones que están detrás de esta mirada, son muy reveladoras de la manera como estos actores claves entienden el proceso de enseñanza aprendizaje y el derecho a la educación de todos los niños (Miranda, 2011, p. 23).

En el caso del Perú, la Directiva № 004-VMGP-2005, aprobada por R.M. № 0234-2005-ED, establece las disposiciones pedagógicas y administrativas para la evaluación de los aprendizajes de los estudiantes en los niveles de inicial, primaria y secundaria. Asimismo, establece las condiciones de promoción, repetición y recuperación. En primaria, los alumnos de primer grado son promovidos al grado superior de forma automática. Los alumnos de $2^{\circ}$, $3^{\circ}, 4^{\circ}, 5^{\circ}$ y $6^{\circ}$ grados que, al término del año escolar, obtienen la nota " $\mathrm{C}$ " en dos áreas curriculares (Lógico Matemática y Comunicación Integral) repiten 134 I el grado automáticamente. En secundaria, repiten los alumnos que, al final 
del año escolar, desaprueban cuatro o más áreas curriculares. Ello incluye las áreas o talleres que fueron creadas como parte de las horas de libre disponibilidad y el área curricular pendiente de subsanación.

Una idea del estado de la repetición se puede apreciar a través del porcentaje de repetidores que, en la última década, ha disminuido en primaria desde 10,7\% en 2000 hasta 6,7\% en 2009, año de la aplicación de la prueba PISA. Esta disminución opera también en secundaria, en donde la repetición disminuye desde $5,2 \%$ hasta $5 \%$ para el mismo periodo. Las cifras referidas a la repetición por cada grado registran valores más altos en los primeros grados $^{6}$. A estas cifras se debe agregar que, según fuentes oficiales, la situación en la zona rural es preocupante, pues hacia el año 2009 en primaria el porcentaje de repetidores llegaba a $27 \%$ y $24 \%$ en segundo y tercer grado, y a $16 \%$ y $14 \%$ en cuarto y quinto grado, respectivamente. Estos porcentajes representan más del doble que las proporciones correspondientes al área urbana. Cabe destacar el hecho de que aun en primer grado -en el que la promoción es automática según la norma nacional- el $11 \%$ repitió el grado en el área rural (Minedu, Edudatos $\left.\mathrm{N}^{\circ} 4\right)$.

\section{La composición social y la escuela}

Al acceder a su escolaridad, los alumnos traen consigo diversas experiencias personales y sociales que se van a manifestar en sus logros de aprendizaje; $y$, por eso, se vuelve importante considerar estos orígenes como un factor presente en la escuela. Este interés por lo que se conviene en llamar la composición social de la escuela data desde hace varias décadas cuando el célebre Informe Coleman (Coleman et ál., 1966) mostró que las características de los compañeros de aula tienen mayor importancia en la variación del logro de los estudiantes de las clases desfavorecidas que cualquier otro factor, como el equipamiento escolar e incluso las actitudes de los docentes. Este informe seminal ha dado lugar a una larga tradición de investigación social sobre las escuelas efectivas, y existe una abundante literatura al respecto.

Haciendo un recuento de los estudios internacionales de la serie PISA, Froemel $(2006$, p. 20) concluye -entre otros puntos- que el efecto que tiene la interacción entre las variables relacionadas con los antecedentes socioeconómicos individuales de los alumnos y los de la escuela a la que asisten sobre el desempeño escolar es mucho más poderoso que aquel solamente atribuible a las variables individuales. Por eso, para este autor, los antecedentes y la composición social serían los mejores predictores del logro.

$6 \quad$ Los datos se encuentran disponibles en Escale (2010). 
Otros estudios recientes que utilizan los resultados de la evaluaciones internacionales (Crahay y Monseur, 2008) ratifican la existencia de los efectos de agregación, producidos sobre el rendimiento escolar como producto de la agrupación de una misma categoría social de alumnos en un mismo centro escolar. Este efecto se refleja en la ubicación de los estudiantes que no siguen su escolaridad en ciertas escuelas de manera aleatoria: aquellos de antecedentes socioeconómicos favorecidos frecuentan las escuelas que tienen estas características y estas son precisamente mejores, porque son frecuentadas por alumnos favorecidos.

En el Perú, los estudios sobre los factores asociados al rendimiento escolar señalan que los estudiantes que asisten a escuelas con un alto nivel socioeconómico promedio tienden a tener mejores rendimientos que aquellos que asisten a escuelas de bajo nivel socioeconómico promedio. Respecto a ello, Miranda anota que:

Cabe señalar que, el nivel socioeconómico y cultural al que pertenece el estudiante no solo explica las diferencias de rendimiento al interior de la escuela, sino también entre escuelas. Además, el porcentaje explicado de la variación del rendimiento entre las escuelas (34\%) es mayor que el porcentaje explicado de la variación del rendimiento entre alumnos (1\%). Así, aun cuando se esperaría lo contrario, estos resultados muestran dos situaciones importantes. Primero, la explicación casi trivial de la desigualdad del rendimiento al interior de las escuelas podría atribuirse a la existencia de pocas diferencias en los antecedentes socioeconómicos y culturales de los estudiantes de una misma escuela, aun cuando estas existan. Segundo, el hecho de que una característica individual explique una alta proporción de las diferencias de rendimientos grupales es indicador de la existencia de diferencias entre escuelas en cuanto al estatus socioeconómico y cultural del alumnado que atienden. En este sentido, los resultados encontrados dan indicios de la existencia de segmentación en el sistema educativo, principalmente, a partir del origen socioeconómico y cultural de los estudiantes ${ }^{7}$ (2008, p. 24).

Por su lado, un estudio sobre la Comprensión de Lectura a partir de una muestra de alumnos de primaria en la ciudad de Lima (Morales, 2011) encuentra que las variables socioculturales y cognitivas que actúan y contribuyen con mayor fuerza al desarrollo de la comprensión de lectura son el vocabulario, la decodificación, la motivación hacia la lectura y el nivel socioeconómico del alumno. En un segundo nivel, también contribuyen el gé-

7 La autora advierte que los porcentajes se refieren a la porción de varianza del rendimiento explicada al incluir únicamente el nivel socioeconómico y cultural del alumno (variable a nivel de individuo). 
nero y la madurez intelectual de los niños mayores. A la evidente influencia de los factores socioculturales sus resultados realzan la importancia de los aspectos motivacionales.

\section{Análisis de resultados}

El Perú ha obtenido un puntaje promedio de 370 puntos en Competencia en Lectura en la evaluación PISA 2009. Los otros países de la OCDE alcanzan un promedio de 498 puntos y los 8 países de América Latina participantes en la encuesta promedian los 408 puntos $^{8}$. A partir de estos resultados, se observa que el país se sitúa en un rango bien modesto en el concierto mundial y latinoamericano. El ranking es desfavorable y hay una distancia de hasta cerca de 200 puntos que separa el Perú de países que han obtenido los puntajes más altos. En lo que respecta a la repetición, los resultados son $71,9 \%$ de alumnos que son no-repetidores y $28,1 \%$ que sí lo son. Una interrogante inmediata es verificar si los alumnos repetidores alcanzan mejores resultados escolares en Competencia Lectora, pues se supone que no debería existir diferencia significativa en los puntajes si la repetición de grado fuera efectiva. Sin embargo, cuando los resultados nacionales son considerados según la repetición, el promedio de lectura es mayor para el no-repetidor y menor para el repetidor. En efecto, los primeros tienen en promedio 394 puntos, y los segundos alcanzan 308 puntos. La diferencia de 86 puntos es sensible entre ambas categorías de alumnos. Además, esta brecha es estadísticamente significativa ${ }^{9}$.

Tabla I

Perú - Resultados promedios en Comprensión Lectora según repetición

\begin{tabular}{cccccccc}
\hline \multicolumn{2}{c}{ Total } & \multicolumn{2}{c}{ No-repetidor } & \multicolumn{2}{c}{ Repetidor } & \multicolumn{2}{c}{ Diferencia } \\
\hline Puntaje & E.E. & Puntaje & E.E. & Puntaje & E.E. & Puntaje & E.E. \\
370,4 & 3,91 & 394,8 & 4.11 & 308,0 & 3,27 & 86,8 & 4,95 \\
\hline
\end{tabular}

NB: Excepto una mención contraria, de aquí en adelante, los resultados de rendimiento son expresados en puntajes promedios. Estos valores son siempre presentados con sus respectivos errores estándares (E.E).

8 Estos países son Colombia, Panamá, México, Chile, Argentina, Brasil y Uruguay.

9 El contraste tiene una diferencia significativa si el cociente obtenido entre la diferencia y el error estándar de la diferencia es mayor que 1,96 a un nivel de confianza $\alpha=0,05$. Su expresión formal es error estándar de la diferencia $\sigma_{\left(\hat{\theta}_{i}-\hat{\theta}_{j}\right)}=\sqrt{\sigma_{\left(\bar{\theta}_{i}\right)}^{2}+\sigma_{\left(\bar{\theta}_{j}\right)}^{2}}$ Así, $86,8 / 4,95=17,5$ es largamente mayor que 1,96 y, entonces, largamente significativo. 
Para facilitar la comparación de este tipo de resultados entre países, la OCDE ha establecido como referencia internacional el valor de 39 puntos en la escala de Comprensión Lectora como el equivalente a la diferencia de un año de escolaridad. En el caso del Perú, este valor ha sido fijado por este organismo en 27,5 puntos de diferencia (OCDE, 2010b, p. 130). Con esta referencia -y según la tabla-, la repetición acumulada en la trayectoria escolar de un alumno peruano repetidor representaría una pérdida equivalente a tres años de escolaridad.

Estos resultados que no incorporan -como se realizará más adelanteotras variables asociadas plantean desde ya serias interrogantes, tanto sobre el rendimiento de los aprendizajes así como sobre la eficacia de la repetición como práctica escolar, sin mencionar las probables consecuencias financieras, sociales y sicológicas que debe engendrar la repetición.

\section{Niveles de Comprensión Lectora y repetición}

Los resultados por niveles de Lectura muestran que la mayoría de los alumnos no alcanza los estándares satisfactorios en competencias básicas de aprendizaje (Ministerio de Educacion, 2010). De los seis niveles clasificados de Competencia en Lectura, el nivel 2, llamado también línea de base, se considera como el nivel básico de competencia en el cual los alumnos empiezan a demostrar la destreza lectora que les permitirá participar de manera efectiva y productiva en la vida social y económica de un país (OCDE, 2010a, p. 13). Con esta línea de base como referencia, hay $64,8 \%$ de alumnos por debajo del nivel 2, y una presencia casi nula en los tres últimos niveles avanzados de la escala. En cuanto a la repetición, los resultados son muy alarmantes en lo que atañe a los repetidores: $89,1 \%$ están por debajo del nivel 2 ; y, luego, desaparecen en los niveles más altos. Muy preocupante es que, en el nivel más modesto (Nivel -1b), los repetidores representan $29,7 \%$ de alumnos. 
Tabla II

Niveles de Comprensión Lectora según repetición

\begin{tabular}{cccccccc}
\hline \multirow{2}{*}{$\begin{array}{c}\text { Comprensión } \\
\text { Lectora }\end{array}$} & $\begin{array}{c}\text { Perú \% } \\
(1)\end{array}$ & \multicolumn{2}{c}{ No-repetidor } & \multicolumn{2}{c}{ Repetidor } & \multicolumn{2}{c}{ Diferencia } \\
\cline { 3 - 8 } & & $\%$ & E.E & $\%$ & E.E & $\%$ & E.E \\
\hline Nivel -1b & 14,1 & 7,8 & 0,72 & 29,7 & 1,82 & $-21,9$ & 1,95 \\
Nivel 1b & 22,0 & 17,7 & 1,22 & 32,5 & 1,82 & $-14,8$ & 2,12 \\
Nivel 1a & 28,7 & 29,2 & 1,36 & 26,8 & 1,98 & 2,4 & $2,40 *$ \\
Nivel 2 & 22,1 & 27,6 & 1,05 & 9,0 & 1,01 & 18,6 & 2,12 \\
Nivel 3 & 10,1 & 13,4 & 1,05 & 1,9 & 0,37 & 11,5 & 1,11 \\
Nivel 4 & 2,6 & 3,6 & 0,69 & & & & \\
Nivel 5 & 0,4 & 0,6 & 0,29 & & & & \\
Nivel 6 & 0,0 & 0,1 & 0,06 & & & & \\
\hline
\end{tabular}

N.B: Todas las diferencias son significativas a $p<0,001$

Fuente: Ministerio de Educación, Unidad de Medición de la Calidad Educativa (2010). El resto de columnas son cálculos propios.

Además de tener solamente $35,2 \%$ de alumnos por encima de la línea de referencia, (100-64,8\%), la situación se vuelve muy sensible para los repetidores, que representarían solamente 10,9\% (100-89,1\%). Este resultado, también, se suma a las dudas sobre los supuestos beneficios de la repetición para mejorar los aprendizajes.

\section{Variaciones de la Comprensión Lectora y la repetición}

Las variaciones del desempeño en Comprensión Lectora se pueden analizar a través de su descomposición entre aquella parte que se explica por las diferencias entre las escuelas y aquella que resulta de las variaciones entre los alumnos. Estos dos componentes se conocen como la varianza intraescuela y la varianza interescuela. Su relación se mide por el coeficiente "rho", llamado también coeficiente de correlación intraclase (ICC), que se formula como:

$$
\rho=\frac{\tau_{00}}{\tau_{00}+\sigma^{2}}
$$


Cuadro 3

Varianzas del rendimiento escolar entre escuelas y alumnos

\begin{tabular}{lcccccc}
\hline \multirow{2}{*}{$\begin{array}{l}\text { Varianzas del } \\
\text { rendimiento }\end{array}$} & $\begin{array}{c}\text { Componente } \\
\text { varianza }\end{array}$ & E. E. & \multicolumn{2}{c}{ No-repetidor } & \multicolumn{2}{c}{ Repetidor } \\
\cline { 4 - 7 } & & & Valor & E.E & Valor & E.E \\
\hline $\begin{array}{l}\text { Varianza entre } \\
\text { escuelas }\end{array}$ & 5128.4 & 171.92 & 4418.0 & 202.45 & 3312.5 & 368.26 \\
$\begin{array}{l}\text { Varianza entre } \\
\text { alumnos }\end{array}$ & 4607.4 & 205.46 & 4330.7 & 260.62 & 4155.5 & 273.83 \\
Rho & 0.54 & 0.01 & 0.50 & 0.02 & 0.44 & 0.03 \\
\hline
\end{tabular}

La varianza entre escuelas representa $54 \%$; y la de los alumnos, $46 \%$. En PISA 2009, estos valores son relativamente altos a escala internacional; y, entre los ocho países latinoamericanos participantes, solamente Panamá y Perú bordean este resultado. Como referencia, los países que lideran la clasificación del rendimiento escolar se sitúan en valores de -aproximadamente- 0,30 .

Esta variabilidad entre escuelas se mantiene en lo que respecta a los no-repetidores $(0,50)$, pero disminuye para los repetidores $(0,44)$. Estos últimos constituirían grupos más homogéneos y, probablemente, se agrupan en escuelas con una composición social también similar. Esta posibilidad será explorada más adelante.

Un fenómeno interesante que se ha señalado en las evaluaciones nacionales es que este coeficiente varía según la progresión del alumno (Miranda, 2008, p.16). Así, mientras se avanza en el ciclo escolar del cuarto grado de primaria hacia quinto de secundaria, la diferencia de rendimiento atribuible a la escuela tiende a disminuir de $46 \%$ a $31 \%$; en consecuencia, las distancias vinculadas a las características individuales de los alumnos se vuelven más importantes. Aunque no se trata de las mismas pruebas (Comprensión Lectora en PISA y Comunicación de acuerdo con el Ministerio de Educación del Perú) ni de los mismos tipos de población de alumnos (evaluación censal y muestral), el valor de rho 0,31 es netamente inferior al valor encontrado aquí $(0,53)$. Probablemente, la explicación -o parte de ella- reside en que PISA hace referencia a una cohorte de quince años, lo cual puede abarcar varios grados, mientras que las pruebas nacionales se dirigen a un grado escolar en particular. Esta interrogante merece contar con mayores evidencias en futuras investigaciones. 


\section{Antecedentes socioeconómicos, Comprensión Lectora y repetición}

Los informes sobre los resultados de PISA 2009 indican que, en la mayoría de países, los efectos del origen social del alumno sobre los logros son un factor muy importante. Esta aserción se basa en la utilización del índice ESCS, considerado como un buen indicador de los antecedentes socioeconómicos, pues incorpora un conjunto de indicadores del contexto familiar del alumno. En este estudio, se utiliza el ESCS para medir la fuerza de la relación entre estos antecedentes y el puntaje obtenido en la prueba de lectura. Este vínculo se muestra con la ecuación lineal que relaciona las dos variables:

$Y_{i j}=V_{00}+\gamma_{10}{ }^{*} E S C S_{i j}+r_{i j}$, en donde figuran la constante, la pendiente $\mathrm{y}$ el valor $r_{i j}$ del error residual respectivamente.

Como es sabido, la pendiente de esta ecuación mide la variación en el puntaje del alumno como producto del cambio en una unidad del índice. Entonces, mientras más inclinada hacia arriba se muestre la pendiente -es decir, mientras más vertical-, el impacto del índice socioeconómico es mayor sobre los resultados escolares y sugiere mayor inequidad. Por el contrario, una gradiente moderada indica un menor impacto del índice, en otras palabras, mayor equidad.

Tabla IV

Rendimiento en Comprensión Lectora según el índice ESCS y la repetición

\begin{tabular}{ccccccc}
\hline \multicolumn{2}{c}{ Comprensión Lectora } & \multicolumn{2}{c}{ No-repetidor } & \multicolumn{2}{c}{ Repetidor } \\
\hline & Puntaje & E.E. & Puntaje & E.E. & Puntaje & E.E. \\
\cline { 2 - 7 } Intercepto & 424,03 & 4,40 & 436,6 & 4,23 & 355,62 & 4,80 \\
ESCS & 40,98 & 2,04 & 37,97 & 2,14 & 25,29 & 2,17 \\
Rho & 27 & 0,03 & 26 & 0,03 & 11 & 0,02 \\
\hline
\end{tabular}

N.B: Todas las diferencias son significativas.

El resultado de la ecuación $Y_{i j}=424+41^{*} E S C S_{i j}+r_{i j}$, muestra un valor de 41 puntos para la pendiente. Este es el valor más alto entre todos los países latinoamericanos y mayor que el promedio de 38 puntos para los países de la OCDE (2012b, p. 55). Ello evidencia la gran importancia de los orígenes sociales que se asocian al rendimiento escolar. Por otra parte, confirmando el hecho precedente, el valor de rho da cuenta de que la proporción del desempeño escolar que se puede atribuir a los antecedentes sociales alcanza el 
27\%. Este valor nuevamente es el más alto entre todos los países latinoamericanos y los países de la OCDE (14\%).

En cuanto a la repetición, un alumno no-repetidor parte con un capital de 436 puntos, es decir, un diferencial de 81 puntos de ventaja frente a su compañero repetidor (355 puntos). A esta ventaja de inicio, se agrega además aquella que otorga el antecedente socioeconómico. En otras palabras, el aumento de un punto en la escala ESCS corresponde a una ventaja de 38 puntos en la escala de Comprensión Lectora, frente a un aumento de solamente 25 puntos para un repetidor. Por otra parte, la varianza de los resultados desciende sensiblemente de $26 \%$ a $11 \%$ entre estos dos grupos, lo cual confirma una menor dispersión de puntajes entre los repetidores y, también, una mayor homogeneidad.

El siguiente gráfico ayuda a ilustrar la lectura de los resultados anteriores. En este, se muestra las rectas que registran la variación de los antecedentes socioeconómicos medidos por el índice ESCS y el logro en lectura, según la repetición.

Comprensión Lectora e índice ESCS por condición de repetición

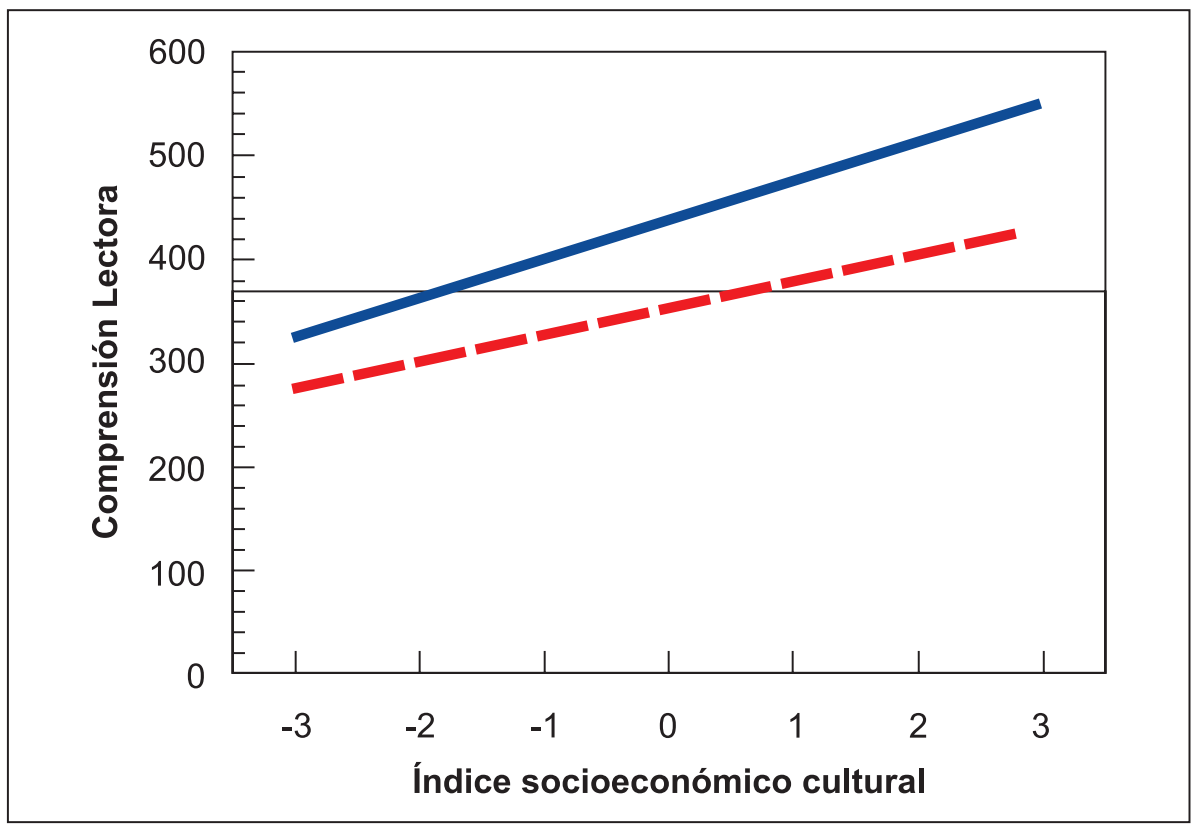

Aquí, se observa claramente que, conforme el índice socioeconómico aumenta, también incrementan los logros para todos los alumnos; ello, cabe 
anotar, ocurre independientemente de la condición de repetición. Sin embargo, también destaca que las rectas presentan distintos puntos de inicio, y el repetidor siempre tiene menores resultados que su compañero no repetidor a lo largo del eje ESCS, es decir, sea cual fuere su antecedente social. La diferente pendiente de las rectas informa que el no-repetidor -que ya viene con ventaja desde la partida- continúa obteniendo mayor ventaja conforme su índice de antecedente social se incrementa.

Otra manera de leer las desigualdades en la Comprensión Lectora, según el indicador ESCS, puede efectuarse a través de la amplitud de la pendiente. En efecto, mientras más extendida es esta, más amplia resulta la dispersión y ello implica la existencia de una población más heterogénea de alumnos. Esta dispersión se mide a través de los cuartiles, que distribuyen al conjunto de alumnos en cuatro proporciones iguales: $25 \%$ de estudiantes para cada una.

Tabla V

Rendimiento en Comprensión Lectora según cuartiles socioeconómicos ESCS y repetición

\begin{tabular}{ccccccc}
\hline \multicolumn{3}{c}{ Comprensión Lectora } & \multicolumn{2}{c}{ No-repetidor } & \multicolumn{2}{c}{ Repetidor } \\
\hline $\begin{array}{c}\text { Cuartiles } \\
\text { ESCS }\end{array}$ & Puntaje & E.E. & Puntaje & E.E. & Puntaje & E.E. \\
\hline Q1 (-2.90) & 302,60 & 3,244 & 330,37 & 3,922 & 272,72 & 5,055 \\
Q2 (-1.77) & 353,94 & 3,097 & 384,62 & 3,637 & 298,01 & 5,289 \\
Q3 (-0.93) & 390,12 & 3,727 & 412,03 & 3,755 & 320,29 & 5,273 \\
Q4 (0.35) & 434,11 & 7,304 & 452,52 & 7,518 & 342,86 & 4,464 \\
\hline
\end{tabular}

N.B: Entre paréntesis, figura el valor de corte correspondiente a cada cuartil. Todas las diferencias entre promedios de esta tabla son estadísticamente significativas.

La amplitud de la dispersión de los puntajes de rendimiento se refleja en el valor del índice ESCS entre el primer y el último cuartil, que va desde -2,90 hasta 0,35 , es decir, un rango bastante amplio de 3,2 puntos en la escala. Este último representa la diferencia que existe entre los alumnos más desfavorecidos (Q1 extremo inferior de 25\%) y los más favorecidos (Q4 extremo superior de $25 \%$ ) en la escala socioeconómica. Ello se traduce en una diferencia de 132 puntos en la Comprensión Lectora, que va desde 302 hasta 434 puntos. Cada punto de este diferencial equivale a 41 puntos, valor ya encontrado en la ecuación de la tabla precedente.

En lo que respecta a la repetición, los alumnos no-repetidores del cuartil superior Q4 llegan a tener 122 puntos más que los alumnos del modesto Q1. Por otro lado, se debe señalar que esta diferencia entre cuartiles es muchísimo menor entre los repetidores, pues solamente alcanza 70 puntos. Esto 
confirmaría el resultado anterior de las pendientes de las rectas, según el cual los repetidores constituyen un grupo con antecedentes socioeconómicos más homogéneos que los alumnos no-repetidores.

\section{El efecto del estanque en la repetición de alumnos}

Parece lógico y normal que, si el criterio de repetidor/no-repetidor se basa en el desempeño escolar del alumno y este es preestablecido a escala nacional, entonces las escuelas con alumnos de alto rendimiento deberían tener menos repetidores que las escuelas con alumnos de bajo rendimiento. Sin embargo, el porcentaje de repetidores en una escuela con alto promedio en el desempeño puede ser tan importante como en una escuela con bajo promedio, sobre todo, donde existen grandes diferencias de logro entre escuelas y es el docente quien generalmente decide la promoción de grado del alumno.

Cabe anotar que, frecuentemente, la decisión de repetir un grado se encuentra a nivel de la escuela y no de la familia. Es la libreta de notas/puntajes de las evaluaciones la que determina la promoción del alumno. No existen procedimientos que involucran a los padres, autoridades y cuerpo docente, y la repetición no se basa en estándares nacionales. A partir de ello, puede ocurrir el llamado "efecto estanque ${ }^{10}$, que es un fenómeno por el cual la repetición del alumno es juzgada en términos relativos en comparación con otros estudiantes de su categoría social o de la categoría social de su escuela, y no en términos de parámetros nacionales (Ikeda, 2005).

Para facilitar la lectura, en este trabajo, se ha construido un gráfico con cuadrantes, que permite presentar una tipología sencilla para observar la dispersión de logros. Estos cuadrantes se abarcan de $A$ hasta $D$, y en ellos figuran los rendimientos promedio de cada una de las 240 escuelas de la muestra y el porcentaje de repetidores en las mismas. Se ha retenido como valores referenciales un valor de 370 puntos, que es el promedio nacional en Comprensión Lectora; y $28 \%$, el porcentaje de repetidores ${ }^{11}$.

Si el fenómeno de "efecto estanque» es pequeño, se puede esperar que haya muchos puntos concentrados en el cuadrante $D$ de la distribución, donde se sitúan los alumnos con bajo rendimiento y con altas repeticiones. Por otra parte, si la distribución se presenta dispersa sin concentración de puntos en particular -como es el caso aquí-, el efecto estanque es mayor. Es decir, hay alumnos repetidores incluso en las escuelas de alto rendimiento; sin

10 Este efecto es mejor conocido bajo el nombre de "frog-pond effect" desde los años ochenta en los anales de la sicología social.

11 La curva es solamente referencial y producto de una ecuación lineal suavizada, que facilita la lectura de la tendencia de los puntos. 
embargo, este efecto de agrupamiento ocurre con mayor frecuencia en las escuelas en las que la mayor parte de alumnos tiene bajo rendimiento.

El cuadrante A contiene a las escuelas con un buen desempeño (mayor que el promedio) y baja repetición (menor que el promedio), y agrupa el $22 \%$ de las escuelas con $24 \%$ de alumnos. La zona B tiene escuelas con alto rendimiento, pero alta proporción de repetidores, que son $23 \%$ y atienden $19 \%$ de alumnos. En el cuadrante $C$, hay $26 \%$ de alumnos en escuelas con bajo desempeño, pero con repetición menor que el promedio nacional -y ellas constituyen el $23 \%$-. Finalmente, en el cuadrante D, se sitúan las escuelas doblemente desfavorecidas, que tienen bajo rendimiento y más repetidores. Sus proporciones se distinguen claramente del resto de escuelas, pues atienden al $30 \%$ de alumnos y representan al $33 \%$ del total de escuelas. El gráfico muestra, además, el contraste evidente entre las zonas $\mathrm{A}$ y $\mathrm{C}$ versus las zonas $B$ y $D$, con la alta dispersión en los promedios de las escuelas en estas zonas.

Porcentajes de repetición y promedios de Comprensión Lectora en las escuelas

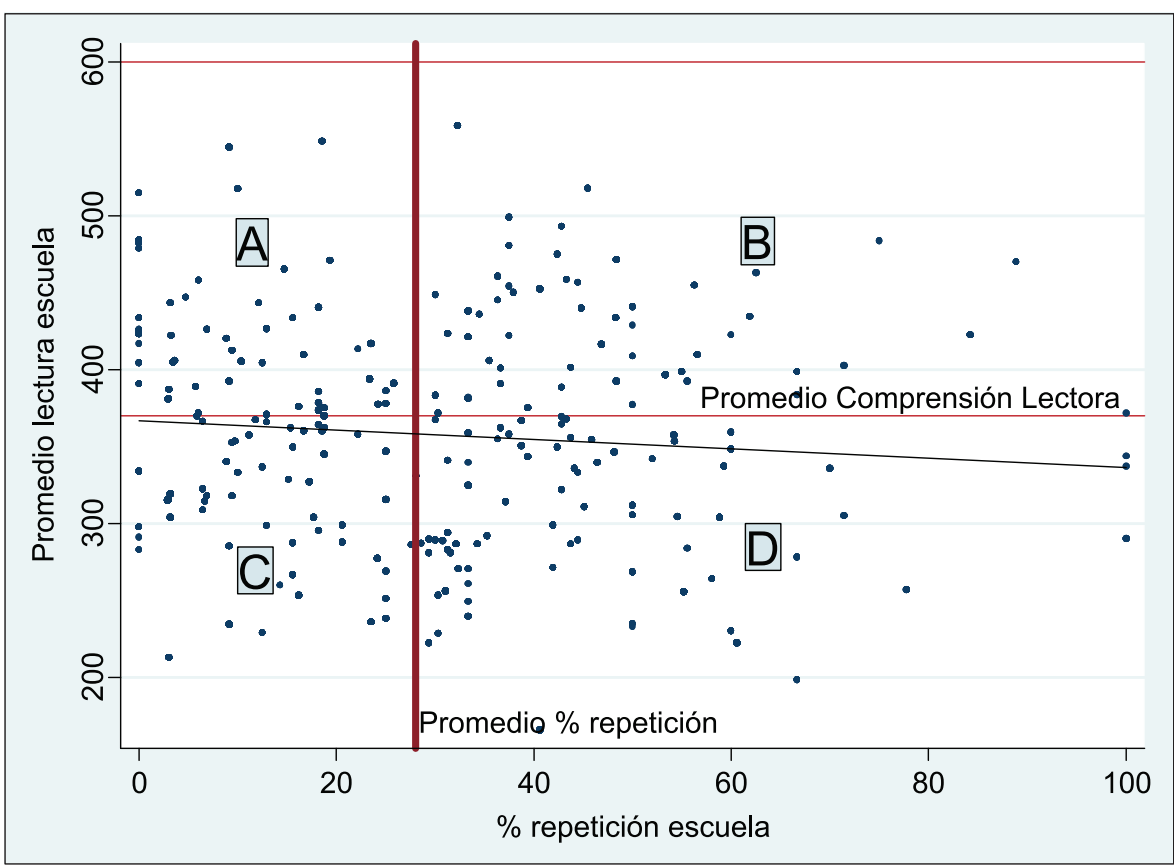

Un análisis suplementario en el que intervienen los cuartiles socioeconómicos permite dar cuenta de la presencia de un efecto de composición. 
En efecto, los alumnos del muy modesto cuartil Q1 representan solamente el $11 \%$ en el cuadrante $A$, mientras que aquellos del muy favorecido cuartil Q4 son el $38 \%$ en el mismo cuadrante. Ocurre lo inverso en el cuadrante $D$, donde los primeros son $50 \%$; y los segundos, solamente $13 \%$.

Este fenómeno de composición social de las escuelas ya ha sido constatado por los estudios sobre la eficacia y la equidad escolar en las evaluaciones anteriores de PISA (Monseur y Crahay, 2008), en los que se corrobora que el beneficio de un alumno de antecedente socioeconómico desfavorecido es mayor si este sigue sus estudios en una escuela favorecida. Por el contrario, el rendimiento será menor si este estudiante desfavorecido acude a una escuela desfavorecida.

\section{Un modelo multinivel de la Comprensión Lectora}

La literatura abunda lo suficiente sobre la fuerte influencia de los antecedentes socioeconómicos de los alumnos sobre los resultados escolares. Menos evidente es la idea de que el contexto social de la institución educativa también puede ejercer una influencia sensible sobre los resultados de los estudiantes. Generalmente, se utiliza el término «composición social» de la escuela para identificar esta probable influencia (Montt, 2012; Willms, 2010).

Para continuar con el análisis de los efectos de la repetición en el rendimiento escolar, se propone un modelo multinivel que incorpore también las variables de antecedentes socioeconómicos y de composición social de la escuela como variables predictoras del rendimiento escolar. Con este propósito, para traducir la textura social de la institución, se ha construido una variable que se llamará convencionalmente Mu_Escuela. Esta equivale al promedio del índice ESCS correspondiente a cada una de las 240 escuelas de la muestra. Para empezar, el modelo de base es el llamado modelo vacío, nulo o incondicional, el mismo que no contiene ninguna variable predictiva y que será luego contrastado con un modelo condicional que incorporará las otras variables. Este último se formula así en las siguientes ecuaciones:

Modelo Nivel-1

$Y_{i j}=b_{0 j}+b_{1 j}{ }^{*}\left(\right.$ ESCS $\left._{i j}\right)+b_{2 j}{ }^{*}\left(\right.$ REPETICION $\left._{i j}\right)+r_{i j}$

Modelo Nivel-2

$B_{0 j}=v_{00}+v_{01}^{*}\left(M U_{-} E S C U E L A_{j}\right)+u_{0 j}$

$B_{1 j}=r_{10}$

$B_{2 j}=r_{20}$ 
Modelo Mixto

$Y_{i j}=\gamma_{00}+\gamma_{01}^{*} M U_{-} E S C U E L A_{j}$

$+\gamma_{10}{ }^{*} E S C S_{i j}$

$+\gamma_{20}{ }^{*}$ REPETICION

$+u_{0 j}+r_{i j}$

Como las variables se han centrado alrededor del promedio a nivel de los alumnos, el coeficiente $\left(v_{01}\right)$ expresa el efecto de la variable Mu_Escuela en el puntaje promedio de la institución educativa y constituye un estimado directo del efecto de composición. El coeficiente a nivel de cada escuela $\left(v_{10}\right)$ para la pendiente de nivel-1 (fija con un mismo valor para todas las escuelas) corresponde al efecto intraescuela.

Por otro lado, el efecto interescuela se obtiene por la suma de ambos. Si la variable agregada es simplemente la adición de los efectos del índice ESCS dentro de cada escuela, entonces los efectos intra e inter serán parecidos y no habrá efectos de composición. Sin embargo, si el coeficiente de la composición es significativamente diferente de cero (es decir, si los coeficientes intra e inter no son iguales), entonces hay una evidencia de la presencia de un efecto de composición que puede ser positivo o negativo (Bryk y Raudenbush, 1992, p. 121).

Cabe anotar que el modelo incondicional -sin la influencia de ninguna variable- reporta un valor rho de 0,54 , que es el mismo encontrado anteriormente en la tabla 3. El modelo 2, que considera únicamente los efectos de la composición social, produce un valor rho de $21 \%$, que disminuye así a la mitad la atribución de la varianza del rendimiento entre escuelas. Esta es evidencia sensible de la importancia que tiene la influencia de la composición social de las escuelas. En consecuencia, una gran proporción de $79 \%$ de resultados escolares en Lectura es explicado por las características de los alumnos. 
Tabla VI

Composición social, antecedentes socioeconómicos y repetición en la Comprensión Lectora

\begin{tabular}{|c|c|c|c|c|c|c|}
\hline & \multicolumn{2}{|c|}{$\begin{array}{c}\text { Modelo } 1 \\
\text { (incondicional) }\end{array}$} & \multicolumn{2}{|c|}{ Modelo 2} & \multicolumn{2}{|c|}{ Modelo 3} \\
\hline & Coef. & (Dev.Es.) & Coef. & (Dev.Es) & Coef. & (Dev.Es.) \\
\hline Intercepto $\tau_{\mathrm{OO}}$ & 5543,54 & $(74,45)$ & 1246,08 & $(35,29)$ & 1099,46 & $(33,15)$ \\
\hline Residuo $\sigma^{2}$ & 4602,77 & $(67,84)$ & 4532,46 & $(67,32)$ & 4242,45 & $(65,13)$ \\
\hline $\begin{array}{l}\text { Intercepto-1 } \beta_{0} \\
\text { Intercepto2 } \gamma_{00}\end{array}$ & 354,8 & 5,60 & 359,72 & 2,95 & 372,84 & 2,88 \\
\hline Mu_Escuela $\gamma_{01}$ & & & 59,22 & 3,24 & 54,56 & 3,19 \\
\hline $\begin{array}{l}\text { ESCS pendiente, } \beta_{1} \\
\text { Intercepto } 2 \gamma_{10}\end{array}$ & & & 10,09 & 1,21 & 8,45 & 1,19 \\
\hline $\begin{array}{l}\text { Repetición } \\
\text { pendiente, } \beta_{2} \\
\text { Intercepto, } \gamma_{20}\end{array}$ & & & & & $-42,53$ & 2,41 \\
\hline Rho & \multicolumn{2}{|c|}{0,54} & \multicolumn{2}{|c|}{0,21} & \multicolumn{2}{|c|}{0,20} \\
\hline
\end{tabular}

NB: Los valores son significativos.

El modelo 3 incorpora esta vez la variable repetición escolar. En este modelo final, el valor del intercepto iguala prácticamente el valor de 370 puntos, que es el promedio nacional de Comprensión Lectora ${ }^{12}$. Es más, la diferencia de varianza entre escuelas no sufre alteración, pues el valor rho se mantiene prácticamente alrededor de $20 \%{ }^{13}$.

En cuanto a los coeficientes, estos indican que, si un alumno asiste a una escuela con una composición social promedio y luego acude a otra escuela que tiene una composición social con un índice mayor de un punto, incrementará su rendimiento en 54,6 puntos. Si este alumno además posee antecedentes sociales más favorables (un punto mayor en la escala ESCS) que el promedio de sus compañeros, se verá también bonificado con un suplemento de 8,45 puntos en su desempeño. En fin, la tabla muestra que la repetición alcanza un valor altamente negativo de $-42,5$ puntos, que afecta el rendimiento escolar de los alumnos*.

12 A diferencia del promedio nacional, no se debe olvidar que el presente valor tiene en cuenta una variable anidada, como es Mu_Escuela.

13 Se debe tener en cuenta que, por construcción y debido a que son continuas, las variables ESCS y Mu_Escuela se alinean con el valor promedio de la muestra, mientras que Repetición por ser variable categórica referencial tiene el valor cero para la categoría no-repetidor. 
En suma, según el modelo presentado, el antecedente socioeconómico del estudiante, la composición social de la escuela y la repetición explican el $80,2 \%$ del total de la varianza de resultados escolares en Comprensión Lectora $[(5543,54-1099,46) / 5543,5)]$. Estos efectos sociales están altamente presentes en las escuelas peruanas y merecen atención.

\section{La repetición como efecto de la composición y antecedentes sociales}

Después de establecer los efectos de múltiples variables independientes en la Comprensión Lectora, el análisis puede dirigirse también a considerar esta vez a la repetición como variable dependiente. Para analizar fenómenos con una métrica categórica binaria -como es el caso de la variable repetición-, se puede recurrir a un modelo de tipo logístico, en el que la probabilidad de repetición de un alumno i en una escuela j está en función del log odds-ratio ${ }^{14}$ y se expresa como: $\mathrm{ij}=\log$ [ij $1-\mathrm{ij}$. Este logaritmo asume el valor 0 cuando la probabilidad de cualquier resultado es 0,5 , y la probabilidad de éxito o de ocurrencia es semejante. El log odds ratio para ser legible es siempre convertido en una probabilidad según la siguiente ecuación:

$$
\mathrm{ij}=1[1+\exp \{-\mathrm{ij}\}]
$$

Los modelos se construyen agregando los predictores elegidos de nivel 1 y nivel 2, centrados alrededor de sus respectivos promedios. Como es usual, se estima primero el modelo incondicional sin predictor a ningún nivel. Luego, a este modelo se le agrega las variables sociales que hemos retenido hasta ahora. La repetición tiene como formulación lo siguiente:

$$
\eta_{\mathrm{ij}}=v_{00}+\nu_{01}{ }^{*} \text { Mu_Escuela }{ }_{\mathrm{j}}+\nu_{10}{ }^{*} \text { ESCS }_{\mathrm{ij}}+\mathrm{u}_{\mathrm{0j}}
$$


Tabla VII

Impacto de la composición social y los antecedente socio económicos sobre la repetición

\begin{tabular}{|c|c|c|c|c|c|c|}
\hline \multirow[b]{2}{*}{ Efecto fijo } & \multicolumn{3}{|c|}{ Modelo incondicional } & \multicolumn{3}{|c|}{ Modelo condicional } \\
\hline & Coef. & E.E. & $t$-ratio & Coef. & E.E. & $t$-ratio \\
\hline $\begin{array}{l}\text { INTRCPT1, } b_{o} \\
\text { INTRCPT2, } v_{00}\end{array}$ & $-0,943$ & 0,088 & $-10,655$ & $-1,036$ & 0,062 & $-16,460$ \\
\hline Mu_Escuela, $v_{01}$ & & & & $-0,718$ & 0,086 & $-8,337$ \\
\hline $\begin{array}{l}\text { Para ESCS } \\
\text { pendiente, } b_{1} \\
\text { INTRCPT } 2, v_{10}\end{array}$ & & & & $-0,212$ & 0,040 & $-5,215$ \\
\hline
\end{tabular}

NB: Los valores son significativos.

Como puede percibirse, todos los coeficientes tienen valores negativos, pues por convención el modelo asigna a los no-repetidores el valor cero y a los repetidores el valor uno ${ }^{15}$. En el modelo, el valor del intercepto indica que un alumno de origen social promedio que frecuenta una escuela cuyos alumnos tienen también un origen social promedio tiene un valor log-odds previsto de repetición de $-1,036$ correspondiente a una probabilidad predictiva de $\phi_{i j}=\exp (-1,03) /[1+\exp (-1,03)]=0,26$ que es semejante al porcentaje obtenido a nivel nacional para la repetición ${ }^{16}$. Sin embargo, si este mismo alumno sobresale del promedio nacional y se ve favorecido por un punto más en la escala del índice de antecedente socioeconómico, tendría una repetición de (log-odds $-1,036-\log$-odds 0,212$)=-1,248$. Ello corresponde a una probabilidad de $\phi_{i j}=\exp (1,248) /(1+\exp (1,248))=0,22$, es decir, una probabilidad de repetición menor. Cuanto más favorecido socialmente es un estudiante, este tiene una menor probabilidad de repetir.

Asimismo, es posible que la composición de la escuela afecte las posibilidades de repetición, incluso después de controlar el efecto individual del índice ESCS. Así, un alumno de origen social promedio, que asiste a una escuela más favorecida en una unidad mayor de su índice de antecedente social, tendrá un valor probable de repetir: $-1,036+(-0,718)=-1,754$ correspondiente a una probabilidad de $\phi_{i j}=\exp (1,754) / 1+(\exp (1,754))=0,15$. Este resultado realza la importancia de la composición social de la escuela, según

15 Un odds ratio de valor mayor que 1 indica que la probabilidad de ocurrencia de la repetición se adscribe al grupo de no-repetidores. Un valor menor de 1 demuestra que ella ocurre con mayor probabilidad en el grupo de repetidores.

16 Se recuerda que los valores de ESCS y Mu_Escuela han sido centrados alrededor de sus respectivos promedios. El no-repetidor tiene un valor de cero referencial por construcción en la ecuación. 
la cual asistir a una escuela más favorecida socialmente protege mejor contra la repetición que el propio antecedente social del alumno: $15 \%$ contra $22 \%$ de probabilidades de repetición.

Entonces, si se agregan los efectos conjugados de ESCS y Mu_Escuela, estos harían que pertenecer a una categoría social favorecida y, además, frecuentar una escuela socialmente favorecida garantiza al alumno menores probabilidades de repetición. Es decir, a una ventaja de antecedente social se agrega una ventaja de composición social en la escuela.

\section{Conclusiones}

Este trabajo pretende explorar la idea de que los logros escolares encuentran una explicación importante en factores tales como la composición social de las escuelas, el antecedente social y la repetición de grado de los alumnos. Para ello, se ha utilizado los datos peruanos del programa internacional PISA 2009. El análisis realizado muestra que, en la prueba de Comprensión Lectora, el logro de los alumnos es modesto y que el nivel satisfactorio de competencias alcanzadas es escaso. Esto ocurre en un contexto escolar en el que la repetición acumulada alcanza a un tercio de la generación de jóvenes de quince años que siguen aún su escolaridad. En todos los casos, se debe destacar que el alumno repetidor siempre tiene menor logro que el no-repetidor.

La varianza del rendimiento entre escuelas aparece relativamente alta en comparación con los estándares internacionales de PISA. Esta varianza es menor cuando se toma en cuenta solamente a los repetidores, probablemente, como efecto de una mayor homogeneidad. El índice ESCS, que mide el antecedente socioeconómico del alumno, se corresponde sistemáticamente con los logros, pues a medida que este aumenta de valor también aumentan los puntajes, y lo hace mayormente en favor del no-repetidor.

El modelo multinivel propuesto en este trabajo, que integra las variables de antecedentes sociales, composición social y repetición para explicar el rendimiento, alcanza un valor alto, pero probablemente es efecto de la influencia de otras variables no cubiertas por el modelo. Lo más interesante a destacar es que la repetición muestra efectos negativos muy importantes sobre el rendimiento escolar y estos efectos se manifiestan en los alumnos de todo antecedente social y en todas las escuelas. Asimismo, el análisis logístico de la repetición muestra que la composición social de los estudiantes de una institución educativa favorecida protege más contra la probabilidad de repetir que el propio índice social personal del alumno. 
Este estudio deja espacio a varias interrogantes que quedan por explorar. Por una parte, los aspectos de retraso y abandono escolar, la ubicación geográfica de las escuelas (rural/urbana), el tipo de gestión (pública/privada), el tamaño de la escuela, las modalidades ofrecidas, el clima escolar, el tipo de pedagogías empleadas, el entorno psicopedagógico de los aprendizajes, las disciplinas evaluadas, son otras tantas dimensiones que pueden resultar fructíferas. Por otra parte, la construcción y uso de índices socioeconómicos, la calidad de la muestra, la pertinencia de los métodos de análisis, entre otros, plantean desafíos teóricos y prácticos para la investigación educativa.

Aunque no se focaliza sobre el problema de la repetición y de los aspectos sociales de la escuela, conviene citar sucintamente las recomendaciones del Consejo Nacional de Educación para prevenir la deserción y la repetición en los grupos socialmente vulnerables: sistemas de detección oportuna de niños y niñas en riesgo de repetición y abandono escolar, institucionalización de estrategias pedagógicas diferenciadas, programas compensatorios de subsidio directo o indirecto, renovación de las escuelas rurales, especialización e incentivo de docentes que trabajen con población vulnerable, mecanismos eficaces de selección de escuelas y docentes de Educación Intercultural Bilingüe (2006, p. 61).

Además de estas recomendaciones, se puede sugeririntervenciones, tales como el control de la transición de grado a grado para reducir o manejar la práctica de la repetición, reducir la población en situación de sobre edad abordando las causas directas del ingreso tardío y la subsecuente repetición por el costo de oportunidad que significa permanecer en la escuela. Asimismo, se puede reforzar los programas compensatorios de ayuda para familias desfavorecidas, de modo que se aliene la heterogeneidad -por lo menos- de las escuelas públicas y se implemente mecanismos de seguimiento.

Los resultados de este estudio invitan a una reflexión sobre la repetición, con el convencimiento de que quedan por identificar y explicar los mecanismos culturales, sociales y económicos que la originan y la acompañan. Las evaluaciones internacionales y nacionales ofrecen informaciones que pueden contribuir a colmar esta agenda siempre inconclusa, como es la controversia sobre la conservación o la eliminación de la repetición. 


\section{Referencias}

Bernard J.M. et ál. (2005). Le redoublement, Mirage de l'école africaine?. Programme d'analyse des systèmes éducatifs de la CONFEMEN. Dakar: Rapport Pasec/Confemen.

Bophy, J. (2006). Grade repetition. París: Unesco / International Institute for Educational Planning.

Consejo Nacional de Educación (2006). Proyecto Educativo Nacional al 2021La educación que queremos para el Perú. Lima: Consejo Nacional de Educación.

Cueto, S. (2007). Las evaluaciones nacionales e internacionales de rendimiento escolar en el Perú: Balance y perspectivas. Investigación, políticas y desarrollo en el Perú (pp. 405-455). Lima: Grade.

Escale (2010). Indicadores - Tendencias. Escale. Estadística de la calidad educativa. Recuperado de http://escale.minedu.gob.pe/tendencias

Froemel, J.E (2006). Los estudios internacionales del rendimiento y los países en vías de desarrollo: participación, resultados y relevancia. Revista de Educación, número extraordinario, 131-152.

García-Huidobro, J.E. (2001). La deserción y el fracaso escolar. En Educación, pobreza y fracaso escolar (pp. 61-69.). Santiago de Chile: Unicef.

Guadalupe, C. y Villanueva, A. (2013). PISA 2009/2000 en América Latina: una relectura de los cambios en el desempeño y su relación con las condiciones sociales. En Apuntes (72), 157-192.

Ikeda, M. (2005). Grade repetition and its effect on performance in Sacmeq countries. Research Conference, SACMEQ/IIEP (pp. 1-30). París: Instituto Internacional de Planeamiento de la Educación.

Llece/Unesco-Orealc (2010). Factores asociados al logro cognitivo de los estudiantes de América Latina y el Caribe. Santiago de Chile: Orealc.

Ministerio de Educación del Perú (2010). Resultados de la evaluación PISA 2009. Lima Unidad de Medición de la Calidad Educativa - Ministerio de Educación.

(2011). Causas del atraso en la culminación de primaria en el área rural. En Edudatos, Lima, junio (4).

Miranda, L. (2008) Factores asociados al rendimiento escolar y sus implicancias para la política educativa del Perú. En M. Benavides (Ed.), Análisis de programas, procesos y resultados educativos en el Perú: contribuciones empíricas para el debate (pp. 11-40). Lima: Grade. 
(2011). Logros y desafíos para garantizar una educación primaria de calidad. Debate - Siteal, noviembre, 8, 18-28.

Montt, G. (2012). Socioeconomic school composition effects on students outcomes (Disertación de PHD). Indiana: University de Notre Dame.

Monseur, C. y Crahay, M. (2008). Composition académique et sociale des établissements, efficacité et inégalités scolaires : une comparaison internationale. Revue Francaise de Pédagogie (RFP), septiembre, 164, 55-65.

Morales, S. (2011). Variables socioculturales y cognitivas en el desarrollo de la Comprensión de Lectura en Lima. Revista Peruana de Investigación Educativa 3, 96-129.

Organisation for Economic Co-operation and Development (OCDE) (2010a). Resultados del Informe PISA 2009: Lo que los estudiantes saben y pueden hacer. Rendimiento de los estudiantes en Lectura, Matemáticas y Ciencias, Volumen I. París: OCDE, Santillana Educación.

(2010b). PISA 2009. Results Overcoming Social Background-Equity in Learning Opportunities and Outcomes, Volumen II. París: OCDE.

(2012a). Equity and Quality in Education Supporting Disadvantaged Students and Schools. París: OCDE.

OCDE.

(2012b). PISA 2009, Technical Report. París:

(s.f.). Database - PISA 2009. OECD Programme for International Studente Assessment (PISA). En: OECD. Better policies for better lives. Recuperado de http://pisa2009.acer.edu.au/

Raudenbush, S.W. y Bryk, A.S. (2002). Hierarchical Linear Models: Applications and Data Analysis Methods. Thousand Oaks, CA: Sage.

Schiefelbein, E. y Wolff, L. (1993). Repetición y rendimiento inadecuado en escuelas primarias de América Latina: magnitudes, causas, relaciones y estrategias. Boletín del Proyecto principal de educación en América Latina y el Caribe, 30, 17-50.

Schiefelbein, E. et ál. (2002), Primary education in Latin America: the unfinished agenda, Inter-American Development Bank-BID, Washington, D.C.: Banco Interamericano de Desarrollo.

Torres, R. M. (2000). Repetición escolar: ¿Falla del alumno o falla del sistema?. La evaluación (pp. 7-40). Buenos Aires: Ediciones Novedades Educativas.

Unidad de Medición de la Calidad Educativa (2012). UMC. Unidad de Medición de la Calidad Educativa. En Ministerio de Educación del Perú. Recuperado de http://www2.minedu.gob.pe/umc 
United Nations Educational, Scientific, and Cultural Organization (Unesco) (2012). EFA Global Monitoring Report. En : Unesco - Education. Recuperado de http://gmr.uis.unesco.org/selectIndicators.aspx

Willms, J.D. (2010). School composition and contextual effects on Student Outcomes. En Teachers College Record, 112(4), 1008-1037. 